\title{
STRUCTURING FACTORS OF THE CUMACEAN COMMUNITIES OF THE CONTINENTAL SHELF OF SOUTHEASTERN BRAZIL
}

\author{
Pedro Abib Cristales and Ana Maria S. Pires-Vanin \\ Instituto Oceanográfico da Universidade de São Paulo \\ (Praça do Oceanográfico, 191, 05508-120 São Paulo, SP, Brasil) \\ *Corresponding author: pedro.cristales@gmail.com
}

http://dx.doi.org/10.1590/S1679-87592014056206202

\section{A B S T R ACT}

Cumaceans are benthic crustaceans distributed worldwide, from intertidal to abyssal plains. They live in sandy and muddy bottoms and can sometimes be found in the water column. Despite their ecological importance as food items for fishes and other organisms and their usefulness as environmental indicators, very few studies have investigated their biodiversity and distribution in Brazil. The present paper reports an ecological study of the cumacean species found on the continental shelf off Santos, southeastern Brazil. It investigates the composition and spatio-temporal structure of the communities, the main environmental factors responsible for the maintenance of that structure and analyzes the effects of the seasonal oceanographic changes on the community descriptors. A grid of 21 stations located between 10 and $100 \mathrm{~m}$ depth was created in winter 2005 and summer 2006 and the sediment samples collected with a $0.1 \mathrm{~m}^{2}$ box corer. Twenty four species were obtained, five of them being the most numerous: Diastylis sexpectinata, D. sympterygiae, Eudorella sp., Oxyurostylis salinoi and Anchistylis notus, the latter highly dominant in summer. Diastylis sympterygiae was characteristic of the shallowest and least saline stations $(<16 \mathrm{~m}$ depth; $\mathrm{S}=33.07-34.2$ ) in both seasons. Of the total of 919 individuals, nearly $70 \%$ occurred in summer. The area was divided into four depth bands and no significant temporal difference was found as regards the density distribution of cumaceans in those bands as between the samples of the two campaigns. There were, however, spatial differences within bands in the summer campaign: the higher values of density, diversity and richness were close to the $50 \mathrm{~m}$ isobath in both campaigns, a fact that could well be attributed to the ongoing effect of the South Atlantic Central Water in this area. The results showed also that depth and the sediment particle size were the main structural factors determining the composition and distribution of the cumacean species.

\section{RESUMO}

Cumáceos são crustáceos peracáridos cosmopolitas que se distribuem desde a zona entre marés até os fundos abissais. Habitam fundos arenosos e lamosos e eventualmente são encontrados no ambiente pelagial. Apesar de sua importância na dieta de diversos organismos, e de serem considerados bons indicadores ambientais, poucos estudos ecológicos foram realizados com o grupo no Brasil, não havendo uma avaliação do papel que desempenham na estruturação e funcionamento do ecossistema bêntico. $\mathrm{O}$ presente trabalho teve como objetivo descrever a composição e a distribuição espaço-temporal das espécies de Cumacea, investigar a existência de comunidades e de sua estrutura, identificar os principais fatores responsáveis pelos agrupamentos encontrados e avaliar o efeito das mudanças sazonais da estrutura oceanográfica sobre os descritores utilizados. Para tanto duas campanhas foram realizadas, no inverno de 2005 e no verão de 2006, em uma grade de 21 estações situadas na plataforma continental sudeste ao largo de Santos, dispostas em quatro faixas de profundidade (entre 10 e $100 \mathrm{~m}$ ) e amostradas por meio de box corer com $0.1 \mathrm{~m}^{2}$ de área amostral. Do total de 24 espécies encontradas, cinco foram as mais abundantes: Diastylis sexpectinata, D. sympterygiae, Eudorella sp., Oxyurostylis salinoi e Anchistylis notus, esta última altamente dominante no verão. Diastylis sympterygiae foi característica das estações rasas $(<16 \mathrm{~m})$ e com salinidades baixas (33.07 - 34.2), tanto no inverno quanto no verão. Do total de 919 indivíduos obtidos pouco mais de $70 \%$ ocorreu no verão. A área foi dividida em quatro faixas de profundidade e, considerando a distribuição da densidade de cumáceos nessas faixas, não foi encontrada diferença temporal significativa entre as amostras das duas campanhas. Entretanto, houve diferença espacial entre as faixas na campanha de verão, sendo os valores mais elevados de densidade, diversidade e riqueza situados próximo à isóbata de $50 \mathrm{~m}$ em ambas as campanhas, podendo ser atribuídos ao efeito contínuo da Água Central do Atlântico Sul sobre essa área. Os resultados mostraram ainda que a profundidade e as características granulométricas do sedimento foram os principais fatores estruturadores das comunidades de Cumacea e determinantes da composição e distribuição das espécies na área.

Descriptors: Cumacea, Distribution, Benthic community structure, Santos, Continental shelf. Descritores: Cumacea, Distribuição, Estrutura de comunidades bênticas, Santos, Plataforma continental. 


\section{INTRODUCTION}

Cumaceans are peracarid crustaceans, mainly marine, distributed worldwide from intertidal to abyssal plains (JONES, 1976). They inhabit sandy and muddy bottoms, and are sometimes found in the pelagic environment (ANGER; VALENTIN, 1976; MACQUART-MOULIN, 1991). They can also occur in association with seaweeds and bryozoans, (ALFONSO et al., 1998; CONRADI et al., 2000) and in whale carcasses and hydrothermal vents (SMITH, 2003; CORBERA; SEGONZAC, 2010). Despite their importance as food item for many organisms (BĂCESCU; QUEIROZ, 1985; SUTHERLAND et al., 2000), and as initial colonizers of dafaunated sediments (SANTOS; SIMON, 1980), their great abundance in some areas and their role as environmental indicators (CORBERA; CARDELL, 1995; SWAILEH; ADELUNG, 1995), few ecological studies have described the importance of environmental parameters in the distribution and composition of cumacean communities (WATLING; GERKEN, 2005; MARTIN et al., 2010). Most studies in Brazil have focused on the taxonomy of the group (e.g. BRUM, 1966; BĂCESCU; PETRESCU, 1990; ROCCATAGLIATA, 1997) and only one has addressed its ecology (SANTOS; PIRES-VANIN, 1999). The lack of specialists seems to be the main cause of the slow progress made as regards the group's taxonomy, and few studies addressing benthic macrofauna in Brazil include cumaceans. In this context, the continental shelf off Santos, situated in the Southeastern Atlantic, has been the object of an interdisciplinary study outlined to evaluate the influence of the estuarine complex of the Baixada Santista on the adjacent continental shelf. The present paper is part of the studies on the benthic fauna and reports the composition and spatio-temporal distribution of the cumacean species on the continental shelf off Santos. The study evaluates the structure of the communities, as well as the interactions between the oceanographic and sedimentary variables on a seasonal scale.

\section{Material and Methods}

\section{Study Area}

The study area is located between Peruíbe and São Sebastião, between $24^{\circ} 19^{\prime} \mathrm{S}, 46^{\circ} 59^{\prime} \mathrm{W}$ and $23^{\circ} 38^{\prime} \mathrm{S}, 45^{\circ} 14^{\prime} \mathrm{W}$, covering an area of $2,402 \mathrm{~km}^{2}$. The sediment is composed of fine and very fine sand down to the 60 meter isobath, with a gradual increase in mud levels towards the external continental shelf (MAHIQUES et al., 1999). Spots and carbonate "belts" also occur between the 100 meter isobath and the continental slope (MAHIQUES et al., 2004). The study area is under the seasonal influence of the cold and saline South Atlantic Central Water (SACW), a bottom water mass that approaches the coast from the slope in summer and retreats to the coastal shelf in autumn. Between November and March, when the prevalence of the wind is east-northeasterly, the SACW moves close to the bottom towards the coast, leading to the oceanward displacement of the less dense Coastal Water and keeping the Tropical Water relatively distant from the coastline (CASTRO; MIRANDA, 1998). With the advance of the SACW cold waters, a seasonal upwelling occurs at mid depths and a great amount of nutrients becomes available in the water column, which increases primary and secondary production (AIDAR et al., 1993; METZLER et al., 1997). From April to October, the prevalence of southerly winds favors the advance of the Tropical Water towards the shelf and the consequent retreat of the SACW (CASTRO; MIRANDA, 1998).

\section{Field Procedures}

Surveys were conducted between August 26 and September 4, 2005 (winter campaign) and between February 13 and 22, 2006 (summer campaign) aboard the N/Oc. "Prof. W. Besnard" of the University of São Paulo. Twenty one stations were sampled between the 10 and 100 meter isobaths (Fig. 1), positioned with a GPS (Global Positioning System) device. At each site, samples were collected using a $0.1 \mathrm{~m}^{2}$ box corer of 40 liter capacity. Samples were collected in duplicate and the sediment column at each sample was divided into three layers: upper (0$2.5 \mathrm{~cm})$, central $(2.5-5.0 \mathrm{~cm})$, and lower $(5.0-15 \mathrm{~cm})$. Thus, on each cruise, 126 samples (21 stations X 2 replicates X 3 layers) were obtained, resulting in a total of 252 samples. Sediment samples were washed on board with water and sieves of 1.0 and $0.5 \mathrm{~mm}$ mesh sizes and the material retained was fixed in $70 \%$ alcohol. In the laboratory, biological samples were examined and organisms identified to species level. Depth, water temperature, and salinity were determined at each sample site using a CTD (Conductivity, Temperature, Depth). An additional sediment sample was taken at each sampling station to determine the granulometry, carbonates (\%) and total organic matter (\%). Particle size analysis was performed using the sieving and pipetting techniques (SUGUIO, 1973). The percentage of organic material present in the sediment was obtained by the method of weighing and burning the material in a muffle (BYERS et al., 1978) and carbonate content was estimated by sample treatment with a $10 \%$ solution of hydrochloric acid (GROSS, 1971). 


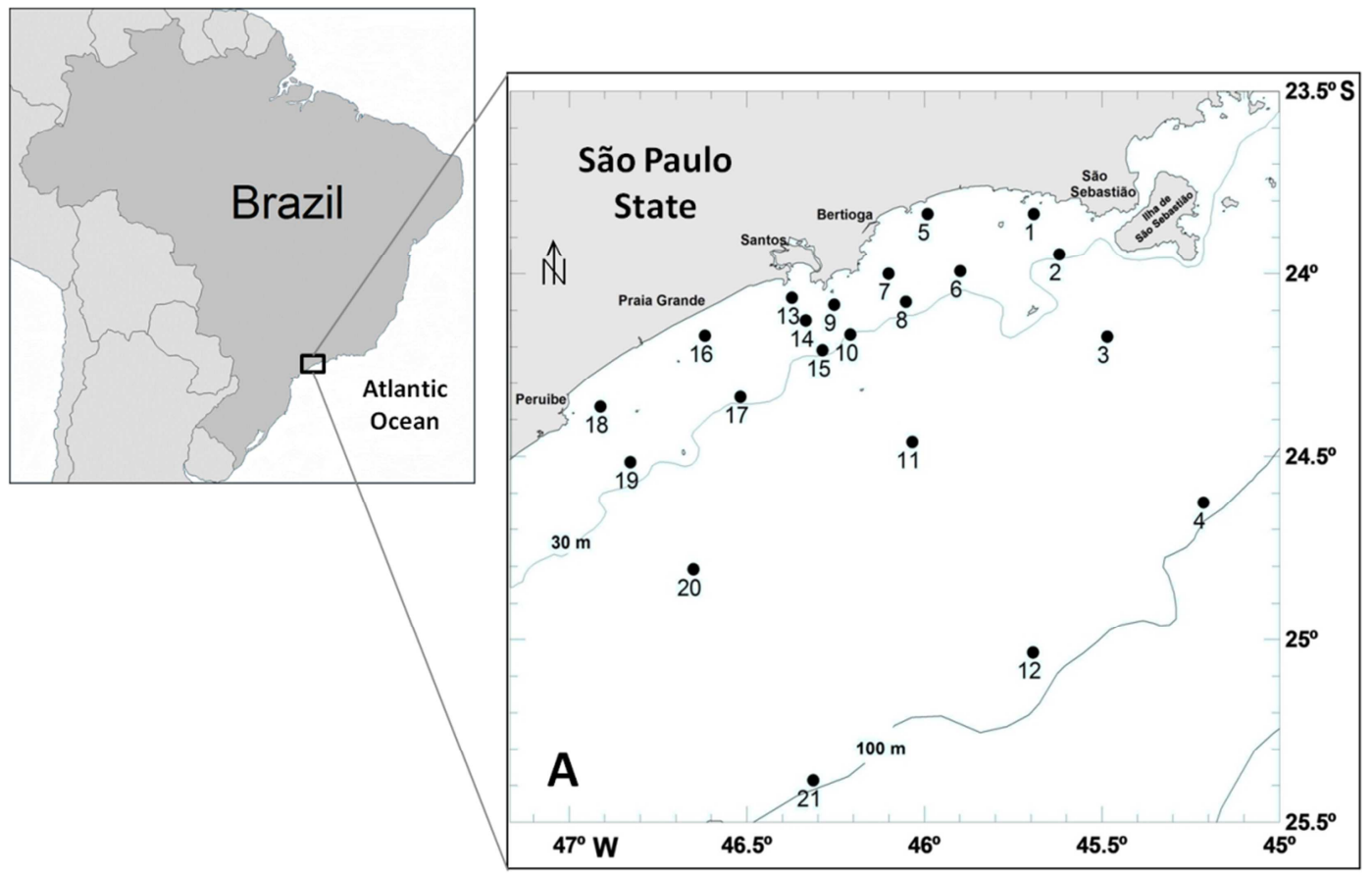

Fig. 1. Map of the continental shelf between Peruíbe and São Sebastião, southeastern Brazilian coast, showing the 21 sampling stations.

\section{Data Analysis}

Replicates were considered separately and not as averages for each sampling season, due to the spotted (scattered, irregular) distribution of both sediments and cumaceans.

Total abundance, frequency of occurrence, and density were calculated for each station. Density was expressed as number of individuals per unit area $\left(0.1 \mathrm{~m}^{2}\right)$. Diversity (H', log base) (SHANNON; WEAVER, 1963), as well as species richness (S) and equitability (J') (PIELOU, 1975) were estimated.

Multivariate analyses were used to identify possible changes in the spatial and temporal structure of the cumacean community. Matrixes with density values were elaborated for this purpose for both sampling campaigns. The Olmstead-Turkey corner test for association (SOKAL; ROHLF, 1995) was used as a cutoff criterion whereby rare species were eliminated. This test is performed by calculating the average between species abundance and frequency, resulting in a graph divided into four quadrants. Each quadrant represents the category of species which are classified into: dominant (abundant and frequent species), local (abundant but not frequent species), constant (very frequent and not abundant species), and rare (not abundant and not frequent species). Eliminating rare species is advisable to avoid skewed distributions (CLARKE; GORLEY, 2006).
After the cutoff, raw data were transformed by fourth root so as to reduce outliers, i.e., to decrease the importance of very high values. After data transformation, the Bray-Curtis similarity coefficient, the most adequate when there is a large number of zeros, was used. On the basis of the resulting triangular matrix, Q-mode hierarchical grouping analyses were carried out for both campaigns.

In addition to these analyses, the SIMPER analysis (Similarity Percentages) was employed to check each species' contribution to the group's formation. Through this analysis, the groups are compared in terms of species abundance and the contribution of each species found in these groups is calculated, which enables the similarity between them to be established (CLARKE; GORLEY, 2006).

The BIOENV analysis was used (CLARKE; AINSWORTH, 1993) for checking which environmental variable combination was better related to the biotic data. To obtain the similarity matrix, the Bray-Curtis similarity coefficient was used for biotic data and the Euclidean distance was used for the environmental data. By using the Spearman weighted correlation method, the matrices were compared and " $\rho$ " rank correlation was calculated through an element by element combination (CLARKE; WARWICK, 2001). The correlation between environmental variables and the cumacean community was also 
applied through Spearman's simple correlation, where a $5 \%$ significance level was considered.

Prior to all the above-mentioned multivariate analyses, an ANOSIM (one-way) similarity analysis was performed to check previously if there was a significant difference between the two sampling periods (summer/winter), and between depth bands within each sampling period. This analysis is highly recommended a priori, since ANOSIM is not a valid test for groups formed by grouping analyses (CLARKE; GORLEY, 2006).

All the analyses described were carried out using the PRIMER v. 6.0 (CLARKE; GORLEY, 2006) and STATISTICA 6 (STATSOFT, 1995) programs.

\section{Results}

The values obtained for the environmental variables analyzed, depth, bottom temperature, salinity, carbonate percentage, and total organic matter are listed in Table 1.

Analyzing the average bottom water temperature in both campaigns, lower values occurred at deeper stations. A string variation was found in summer, while during winter, temperatures were more stable, around $21^{\circ} \mathrm{C}$, except for deep stations 4,12 , and 21 , where temperatures ranged between 16 and $17^{\circ} \mathrm{C}$. Regarding bottom water salinity, stations closer to the coast presented lower values, especially in summer and at places under the influence of the Peruíbe River waters (salinity equal to 33.07 and 33.82 at stations 18 and 16 , respectively). As with temperature, salinity showed greater variability in the summer samples.
Based on the temperature and salinity vertical profile at each station, the presence of the South Atlantic Central Water (SACW) was identified in the first sampling campaign $\left(\mathrm{T}<20^{\circ} \mathrm{C}\right.$ and $\left.\mathrm{S}<36.40\right)$ and, in the second campaign, three water masses were present: South Atlantic Central Water (SACW), Coastal Water $(\mathrm{CW})\left(\mathrm{T}<26^{\circ} \mathrm{C}\right.$ and $\left.\mathrm{S}<34\right)$, and Tropical Water (TW) $\left(\mathrm{T}>20^{\circ} \mathrm{C}\right.$ and $\left.\mathrm{S}>36.40\right)$ (Table 1). During the winter, SACW was identified only at the deepest stations $(4,12$, and 21$)$, all located near the 100 meter isobath. In the rest of the area, the water column was almost homogeneous and occupied predominantly by Coastal Water. In this season a midwater thermocline was observed at the outer stations, where it is present all the year round.

In contrast to the winter campaign, in summer the temperature of the bottom layer was 8 to $10^{\circ} \mathrm{C}$ lower than at the water surface. In the coastal stations up to $30 \mathrm{~m}$ depth the entire water column was homogeneous; from $30 \mathrm{~m}$ depth down to the outer shelf a well-defined thermocline was present at intermediate depths and the water column remained stratified throughout the sampling period. The SACW could be detected over (in) the bottom layer at station 15 and at those stations situated below $50 \mathrm{~m}$ depth. At the other stations on the $30 \mathrm{~m}$ isobath $(2,8,10,17,19)$ a mixture of SACW and CW could be found, with bottom water temperatures varying between 19.08 and 20.65 .

Most stations on the continental shelf, in both winter and summer, had a high sand content (Fig. 2). The deepest stations, 12 and 21, showed remarkable percentages of silt and clay in both campaigns, together with high percentages of organic matter and carbonate with biodetritus.

Table 1. Depth, coordinates and environmental bottom characteristics of the stations sampled in the winter (2005) and summer (2006) campaigns. T: Temperature. O.M.: Organic Matter. Carb.: Carbonate.

\begin{tabular}{|c|c|c|c|c|c|c|c|c|c|c|c|c|c|c|}
\hline \multirow[b]{2}{*}{ Stations } & \multicolumn{7}{|c|}{ Winter (2005) } & \multicolumn{7}{|c|}{ Summer (2006) } \\
\hline & Depth (m) & Latitude & Longitude & $\mathrm{T}\left({ }^{\circ} \mathrm{C}\right)$ & Salinity & O.M. (\%) & Carb. (\%) & Depth (m) & Latitude & Longitude & $\mathrm{T}\left({ }^{\circ} \mathrm{C}\right)$ & Salinity & O.M. (\%) & Carb. (\%) \\
\hline 1 & 20.09 & $23^{\circ} 59^{\prime} .58$ & $045^{\circ} 53^{\prime} .94$ & 21.63 & 34.64 & 1.76 & 5.29 & 19.14 & $25^{\circ} 23^{\prime} .11$ & $046^{\circ} 18^{\prime} .99$ & 25.76 & 34.47 & 1.16 & 4.30 \\
\hline 2 & 34.04 & $23^{\circ} 50^{\prime} \cdot 16$ & $045^{\circ} 59^{\prime} .46$ & 21.42 & 34.72 & & 9.90 & 33.54 & $24^{\circ} 48^{\prime} \cdot 60$ & $046^{\circ} 39^{\prime} .18$ & 19.71 & 35.69 & 5.47 & 4.60 \\
\hline 3 & 58.32 & $24^{\circ} 00^{\prime} .01$ & $046^{\circ} 06^{\prime} .05$ & 20.61 & 35.24 & 3.67 & 8.68 & 57.24 & $24^{\circ} 30^{\prime} .94$ & $046^{\circ} 49^{\prime} .65$ & 16.83 & 34.93 & 2.74 & 9.40 \\
\hline 4 & 85.14 & $24^{\circ} 04^{\prime} .62$ & $046^{\circ} 03^{\prime} .12$ & 17.42 & 35.87 & 2.31 & 19.76 & 84.58 & $24^{\circ} 21^{\prime} .95$ & $046^{\circ} 54^{\prime} .73$ & 15.00 & 35.48 & 2.82 & 18.50 \\
\hline 5 & 15.08 & $24^{\circ} 05^{\prime} \cdot 10$ & $046^{\circ} 15^{\prime} .24$ & 21.23 & 34.64 & 1.22 & 4.80 & 14.48 & $24^{\circ} 20^{\prime} .28$ & $046^{\circ} 31^{\prime} .08$ & 26.10 & 34.18 & 4.01 & 4.50 \\
\hline 6 & 27.40 & $24^{\circ} 09^{\prime} .97$ & $046^{\circ} 12^{\prime} .51$ & 20.92 & 35.71 & 1.10 & 2.30 & 27.40 & $24^{\circ} 10^{\prime} .13$ & $046^{\circ} 37^{\prime} .03$ & 20.93 & 35.71 & 8.54 & 3.00 \\
\hline 7 & 19.15 & $24^{\circ} 12^{\prime} .54$ & $046^{\circ} 17^{\prime} .22$ & 21.16 & 34.73 & 2.34 & 3.10 & 20.11 & $24^{\circ} 03^{\prime} .96$ & $046^{\circ} 22^{\prime} .38$ & 24.88 & 34.67 & 5.29 & 2.20 \\
\hline 8 & 27.14 & $24^{\circ} 07^{\prime} .68$ & $046^{\circ} 20^{\prime} .04$ & 21.15 & 35.06 & 0.95 & 6.40 & 28.16 & $24^{\circ} 07^{\prime} .68$ & $046^{\circ} 20^{\prime} .04$ & 19.60 & 35.69 & 0.93 & 6.30 \\
\hline 9 & 24.07 & $24^{\circ} 03^{\prime} .96$ & $046^{\circ} 22^{\prime} .38$ & 21.08 & 34.93 & 1.62 & 2.80 & 24.64 & $24^{\circ} 12^{\prime} .74$ & $046^{\circ} 17^{\prime} .09$ & 21.51 & 35.84 & 6.16 & 3.20 \\
\hline 10 & 31.20 & $24^{\circ} 10^{\prime} .17$ & $046^{\circ} 37^{\prime} .08$ & 20.83 & 35.16 & 0.84 & 5.86 & 31.22 & $24^{\circ} 05^{\prime} .07$ & $046^{\circ} 15^{\prime} .25$ & 19.08 & 35.74 & 1.04 & 5.13 \\
\hline 11 & 53.61 & $24^{\circ} 20^{\prime} .28$ & $046^{\circ} 31^{\prime} .08$ & 21.09 & 35.86 & 2.02 & 20.44 & 56.18 & $24^{\circ} 09^{\prime} .90$ & $046^{\circ} 12^{\prime} .48$ & 17.17 & 34.43 & 2.69 & 15.62 \\
\hline 12 & 93.48 & $24^{\circ} 21^{\prime} .87$ & $046^{\circ} 54^{\prime} .73$ & 17.13 & 35.83 & 8.96 & 33.17 & 93.29 & $24^{\circ} 00^{\prime} .00$ & $046^{\circ} 06^{\prime} .00$ & 16.49 & 35.71 & 4.56 & 2.65 \\
\hline 13 & 16.23 & $24^{\circ} 30^{\prime} .96$ & $046^{\circ} 49^{\prime} .68$ & 21.10 & 34.95 & 2.20 & 3.00 & & $24^{\circ} 04^{\prime} .61$ & $046^{\circ} 03^{\prime} .08$ & 25.96 & 34.07 & 0.56 & 2.99 \\
\hline 14 & 26.26 & $24^{\circ} 48^{\prime} .49$ & $046^{\circ} 39^{\prime} .02$ & 21.00 & 35.12 & 0.91 & 3.00 & 24.64 & $23^{\circ} 59^{\prime} .58$ & $045^{\circ} 53^{\prime} .94$ & 22.21 & 35.80 & 1.03 & 3.70 \\
\hline 15 & 31.60 & $25^{\circ} 23^{\prime} \cdot 16$ & $046^{\circ} 18^{\prime} .78$ & 20.72 & 35.18 & & & & $23^{\circ} 50^{\prime} .16$ & $045^{\circ} 59^{\prime} .46$ & 18.61 & 35.70 & 1.08 & 5.10 \\
\hline 16 & 13.47 & $25^{\circ} 02^{\prime} .14$ & $045^{\circ} 41^{\prime} .65$ & 21.02 & 34.87 & 1.09 & 3.90 & 14.14 & $23^{\circ} 50^{\prime} .13$ & $045^{\circ} 41^{\prime} .47$ & 26.33 & 33.82 & 2.22 & 4.50 \\
\hline 17 & & $24^{\circ} 37^{\prime} .62$ & $045^{\circ} 12^{\prime} .84$ & 20.51 & 35.05 & 1.24 & & 30.09 & $23^{\circ} 56^{\prime} .82$ & $045^{\circ} 37^{\prime} .20$ & 20.07 & 35.86 & 7.26 & 4.15 \\
\hline 18 & 15.29 & $24^{\circ} 27^{\circ} .66$ & $046^{\circ} 02^{\prime} .04$ & 20.97 & 34.64 & 1.04 & 2.35 & 14.51 & $24^{\circ} 10^{\prime} .32$ & $045^{\circ} 29^{\prime} .10$ & 26.84 & 33.07 & 1.02 & 3.99 \\
\hline 19 & 27.38 & $24^{\circ} 10^{\circ} .32$ & $045^{\circ} 29^{\prime} .10$ & 20.92 & 34.63 & 2.38 & 3.41 & 28.56 & $24^{\circ} 37^{\circ} .70$ & $045^{\circ} 12^{\prime} .88$ & 20.65 & 35.85 & 1.47 & 9.30 \\
\hline 20 & 49.68 & $23^{\circ} 50^{\prime} .16$ & $045^{\circ} 41^{\prime} .52$ & 21.02 & 35.81 & 1.51 & 9.03 & 49.54 & $25^{\circ} 02^{\prime} .16$ & $045^{\circ} 41^{\prime} .64$ & 17.22 & 35.71 & 1.16 & 4.30 \\
\hline 21 & 97.07 & $23^{\circ} 56^{\prime} .82$ & $045^{\circ} 37^{\prime} .20$ & 16.65 & 35.76 & 6.78 & 15.3 & 96.24 & $24^{\circ} 27^{\prime} .60$ & $046^{\circ} 02^{\prime} .02$ & 17.00 & 35.48 & 5.47 & 4.60 \\
\hline
\end{tabular}



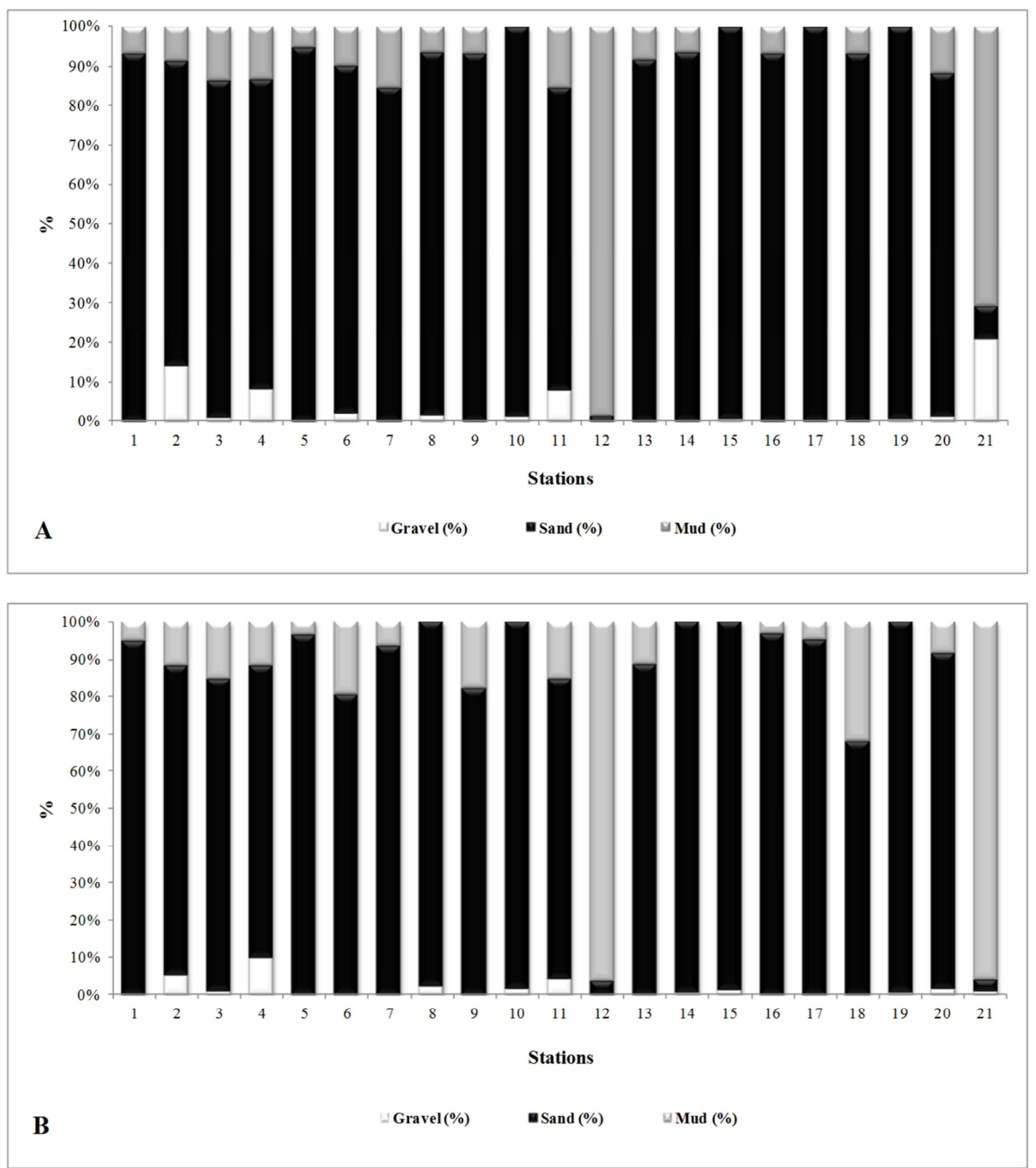

Fig. 2. Distribution of particle size classes (gravel, sand and mud) of the stations sampled on the continental shelf between São Sebastião and Peruíbe. A: Winter survey (2005); B: Summer survey (2006).

\section{Fauna Abundance}

A total of 919 specimens were identified, 246 in the winter and 673 in the summer, distributed in 24 species and four families. Diastylidae was the most specious and numerous, with 7 species and contributing with $68 \%$ of the total number of individuals. Bodotriidae were represented by 6 species, accounting for $18 \%$ of the total abundance.
Leuconidae and Nannastacidae presented 4 and 7 species, respectively, and both contributed with $7 \%$. Of the total species found, 12 were not described (Table 2).

Of the most abundant species found on the winter/2005 campaign, Diastylis sexpectinata contributed $15 \%$ of the total number of individuals, Eudorella sp. 13\%, Diastylis sympterygiae 12\%, and Anchistylis notus and Oxyurostylis salinoi $11 \%$ each. 
In the summer/2006 campaign, Anchistylis notus was much more abundant than the other species, accounting for $54 \%$ of the total individuals. Among the other species were Cyclaspis variabilis with $7 \%$, Diastylis sympterygiae with $7 \%$, Oxyurostylis salinoi with 6\%, and Cyclaspis reticulata with 5\%.

Generally, densities were lower in the winter, with an average of $5.9 \pm 11.2$ ind./ $/ 0.1 \mathrm{~m}^{2}$. Station 3A had a much higher number $\left(68\right.$ ind. $\left./ 0.1 \mathrm{~m}^{2}\right)$ due to the presence of two species with similar abundances, Eudorella sp. (31 ind./0.1 $\mathrm{m}^{2}$ ) and Diastylis sexpectinata $\left(27\right.$ ind./ $\left./ 0.1 \mathrm{~m}^{2}\right)$. At stations 5 , 13, 18, and 21, there were no cumaceans (Fig. 3A). In the summer campaign, total density was nearly 3 times higher than during the previous campaign, and the average density found was $16 \pm 35.17$ ind. $/ 0.1 \mathrm{~m}^{2}$. Variability among samples in this survey was high. The occurrence of high densities at stations 20A (113 ind./0.1 $\left.\mathrm{m}^{2}\right)$ and 20B (206 ind./0.1 $\left.\mathrm{m}^{2}\right)$ was remarkable, and Anchistylis notus was highly dominant at these stations (94 ind. $/ 0.1 \mathrm{~m}^{2}$ and 184 ind. $/ 0.1 \mathrm{~m}^{2}$, respectively) (Fig. 3B). Cumaceans were rare at station 12 ( 1 individual in each sampling) and totally absent at station 21 .

Cumaceans presented a marked decline in abundance with the increase in sedimentary column depth with up to $90 \%$ of specimens occurring in the upper layer $(0-2.5 \mathrm{~cm})$. Considering both campaigns, ten species $(41 \%)$ occurred exclusively in the upper layer and only 4 species (16\%) in all three layers. These species were Anchistylis notus, Oxyurostylis salinoi, Eudorella sp. and Cumella sp. A.

In accordance with Olmstead \& Turkey's graphic representation, most species found on both campaigns were considered rare. Dominant, local, and common species are listed below in order of decreasing numerical importance. During the winter campaign, 11 species were considered rare (Cyclaspis n. sp. A, Leptocuma kinbergii, Cyclaspis sp., Diastylis fabrizioi, Diastylis sp., Leuconidae A, Leuconidae B, Campylaspis sp. A, Campylaspis sp. B, Campylaspis sp. C and Campylaspis sp. D), 1 common (Leucon (Crymoleucon) sp.) and 2 local (Diastylis sexpectinata and Eudorella sp.). In the summer campaign, 12 species were considered rare (Leptocuma kinbergii, Diastylis fabrizioi, Diastylis araruamae, Diastylis sp., Campylaspis sp. A, Campylaspis sp. B, Campylaspis sp. C, Campylaspis sp. D, Campylaspis alveolata, Cumella sp. and Nannastacidae), 2 local (Leucon (Crymoleucon) sp. and Eudorella sp.), and 1 common (Cyclaspis pustulata). The distribution of the number of species by category of dominance in both campaigns is presented in Figure 4.

Table 2. Frequency of occurrence (FO) and total abundance (TA) of cumacean species collected during Winter 2005 (W) and Summer 2006 (S) surveys.

\begin{tabular}{|c|c|c|c|c|}
\hline Species & Survey & Stations & F.O $0(\%)$ & TA \\
\hline \multicolumn{5}{|l|}{ Bodotriidae } \\
\hline Cyclaspis reticulata & w/s & $1,10,15,17,19(\mathrm{~W}) / 1,5,6,7,8,10,13,14,16,17,19,20(\mathrm{~S})$ & $17(\mathrm{~W}) / 36(\mathrm{~S})$ & $16(\mathrm{~W}) 31(\mathrm{~S})$ \\
\hline Cyclaspis variabilis & w/s & $2,3(\mathrm{~W}) 2,6,8,10,14,15(\mathrm{~S})$ & $10(\mathrm{~W}) / 21(\mathrm{~S})$ & $18(\mathrm{~W}) 50$ (S) \\
\hline Cyclaspis pustulata & $\mathrm{s}$ & $1,14,16,18$ & 14 & 8 \\
\hline Cyclaspis sp. A & w/s & $3,11(\mathrm{~W}) 3,11,20(\mathrm{~S})$ & 7 (W) 12 (S) & $3(\mathrm{~W}) / 11(\mathrm{~S})$ \\
\hline Leptocuma kinbergii & w/s & $8(\mathrm{~W}) 8(\mathrm{~S})$ & 2 (W) 2 (S) & $3(\mathrm{~W}) 1(\mathrm{~S})$ \\
\hline Cyclaspis sp. B & w/s & $7,8,16,17,19(\mathrm{~W}) 1,5,7,13,14,16,18(\mathrm{~S})$ & $14(\mathrm{~W}) / 21(\mathrm{~S})$ & $8(\mathrm{~W}) 22(\mathrm{~S})$ \\
\hline Cyclaspis sp. & w & 10,20 & 5 & 2 \\
\hline \multicolumn{5}{|l|}{ Diastylidae } \\
\hline Anchistylis notus & w/s & $1,2,10,11,15,20(\mathrm{~W}) 2,3,6,8,10,11,14,15,17,20$ (S) & $19(\mathrm{~W}) 40$ (S) & $27(\mathrm{~W}) 363(\mathrm{~S})$ \\
\hline Diastylis fabrizioi & w/s & $2,11(\mathrm{~W}) 2(\mathrm{~S})$ & $5(\mathrm{~W}) 5(\mathrm{~S})$ & $2(\mathrm{~W}) 7(\mathrm{~S})$ \\
\hline Oxyurostylis salinoi & w/s & $2,6,8,10,14,15,17(\mathrm{~W}) / 3,6,9,14,15,17,19(\mathrm{~S})$ & $21(\mathrm{~W} / 24(\mathrm{~S})$ & $26(\mathrm{~W}) / 43(\mathrm{~S})$ \\
\hline Diastylis sympterygiae & w/s & $3,8,9,14,16,17,19,20(\mathrm{~W}) / 1,5,7,9,13,16,18(\mathrm{~S})$ & $26(\mathrm{~W}) 26(\mathrm{~S})$ & $30(\mathrm{~W}) 50(\mathrm{~S})$ \\
\hline Diastylis sp. & ws & 3 (W)/11 (S) & 2 (W) 2 (S) & $1(\mathrm{~W}) 1(\mathrm{~S})$ \\
\hline Diastylis araruamae & $\mathrm{s}$ & 11 & 2 & 4 \\
\hline Diastylis sexpectinata & w/s & $3(\mathrm{~W}) / 2,6,11,20(\mathrm{~S})$ & $5(\mathrm{~W}) 14(\mathrm{~S})$ & $38(\mathrm{~W}) 17(\mathrm{~S})$ \\
\hline Diastylidae & w/s & $3,11,16,17,18(\mathrm{~W}) 2(\mathrm{~S})$ & $17(\mathrm{~W}) 2(\mathrm{~S})$ & 12 (W) $11(\mathrm{~S})$ \\
\hline \multicolumn{5}{|l|}{ Leuconidae } \\
\hline Eudorella sp. & w/s & $3(\mathrm{~W}) 3,20(\mathrm{~S})$ & $2(\mathrm{~W}) 10(\mathrm{~S})$ & $31(\mathrm{~W}) 13(\mathrm{~S})$ \\
\hline Leucon (Crymoleucon) sp. & w/s & $3,11,20(\mathrm{~W}) / 3,11,20(\mathrm{~S})$ & $10(\mathrm{~W} / 10(\mathrm{~S})$ & $4(\mathrm{~W}) 11$ (S) \\
\hline Leuconidae A & w & 3,20 & 5 & 2 \\
\hline Leuconidae B & w & 12 & 2 & 1 \\
\hline \multicolumn{5}{|l|}{ Nannastacidae } \\
\hline Cumella sp. A & w/s & $3,4,11(\mathrm{~W}) 3,4,11,20(\mathrm{~S})$ & $12(\mathrm{~W} / 17(\mathrm{~S})$ & $17(\mathrm{~W}) / 26(\mathrm{~S})$ \\
\hline Campylaspis sp. A & w/s & $11(\mathrm{~W}) 1,15(\mathrm{~S})$ & 2 (W) 5 (S) & $1(\mathrm{~W}) 4(\mathrm{~S})$ \\
\hline Campylaspis sp. B & $\mathrm{w} / \mathrm{s}$ & $4(\mathrm{~W}) 4(\mathrm{~S})$ & $2(\mathrm{~W}) / 2(\mathrm{~S})$ & $2(\mathrm{~W}) 3(\mathrm{~S})$ \\
\hline Campylaspis alveolata & $\mathrm{s}$ & 1 & 2 & 2 \\
\hline Campylaspis sp. C & w/s & $4(\mathrm{~W}) 4(\mathrm{~S})$ & $2(\mathrm{~W}) / 2(\mathrm{~S})$ & $1(\mathrm{~W}) 1(\mathrm{~S})$ \\
\hline Campylaspis sp. D & w/s & $20(\mathrm{~W}) 11(\mathrm{~S})$ & $2(\mathrm{~W}) 2(\mathrm{~S})$ & $1(\mathrm{~W}) 1(\mathrm{~S})$ \\
\hline Cumella sp. & $\mathrm{s}$ & 4 & 2 & 2 \\
\hline Nannastacidae sp. & $\mathrm{s}$ & 4 & 2 & 1 \\
\hline
\end{tabular}




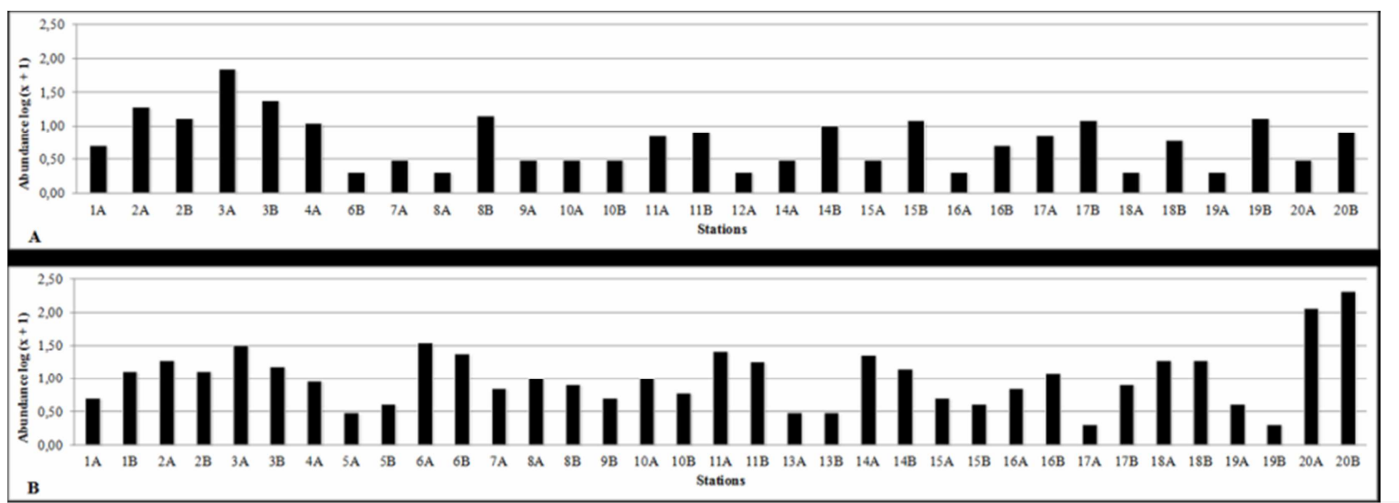

Fig. 3. Cumacea density in the stations sampled on the continental shelf of Santos. A: Winter survey (2005); B: Summer survey (2006).
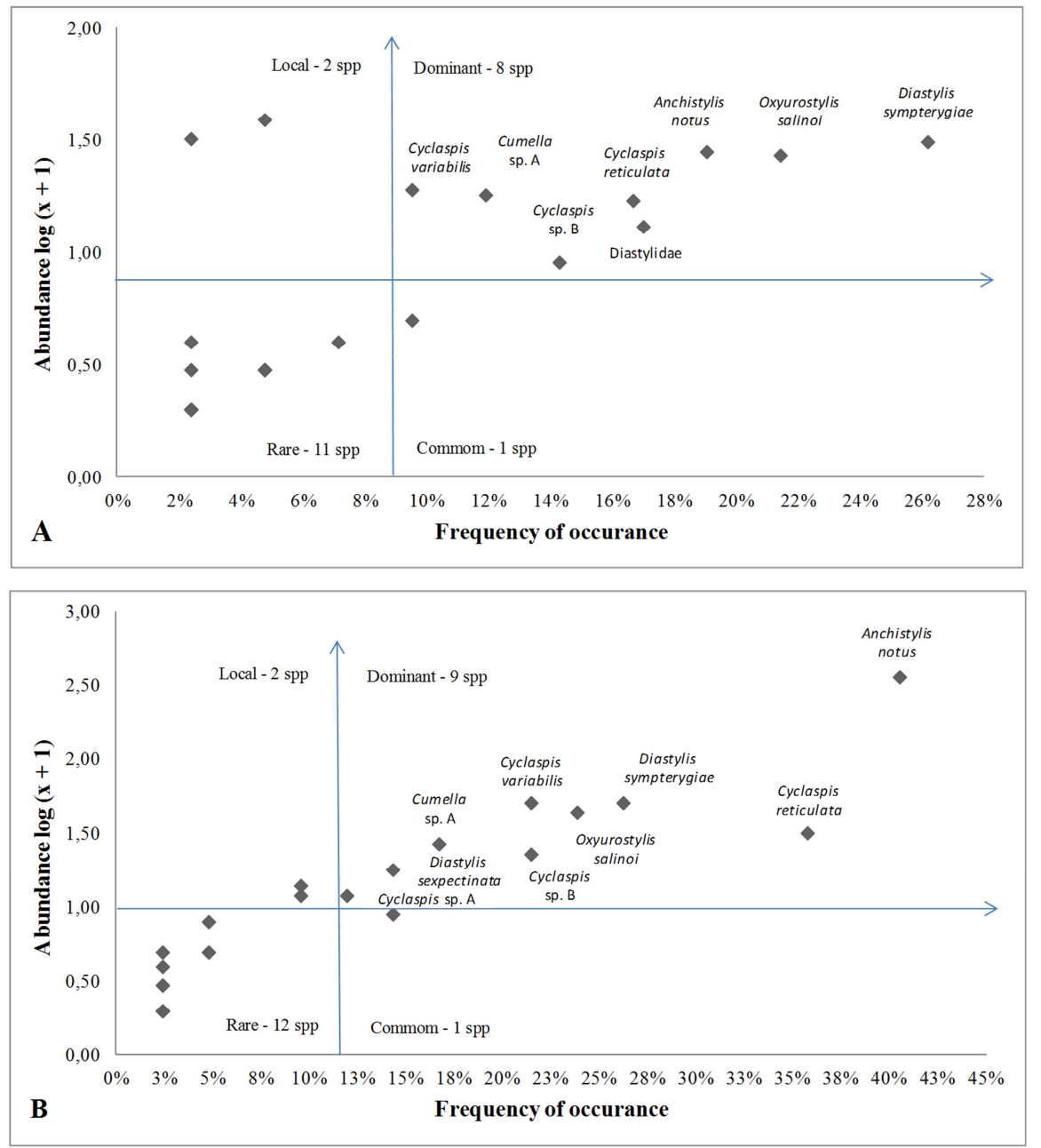

Fig. 4. Plot of the cumacean species abundance, in $\log (x+1)$, in function of their frequency of occurrence in the study area. A: Winter survey (2005); B: Summer survey (2006). 
Community Structure

The ANOSIM similarity analysis was carried out a priori to check the existence of temporal (winter versus summer) and spatial differences (four depth bands). Despite mean density's being nearly three times higher in summer, the results showed that there was no significant temporal difference between density of cumaceans on the two campaigns; the "R" value obtained was not significant (0.015) and the "p" significance level was high (11.5\%). Nor was there any significant spatial difference in cumacean densities in the winter samples (Global $\mathrm{R}=0.299, \mathrm{p}<5 \%$ ), but a significant difference was found in summer (Global $\mathrm{R}=0.064, \mathrm{p}<5 \%$ ) between all the depth bands (Table 3).

The cluster analysis for the winter campaign revealed four main groups. There was no grouping by depth interval but rather a mosaic among the stations, confirming the results obtained by the ANOSIM analysis. Four groups were formed at $30 \%$ similarity level (Fig. 5) and each species' contribution to such groups was evidenced by the SIMPER analysis. The main structural and biotic characteristics of the groups are presented in Table 4.

One group was identified in shallower coastal waters (group A), one in the central area in front of Santos Bay plus station 1 (group B), one at 50 $\mathrm{m}$ depth plus station 2 (group $\mathrm{C}$ ) and the fourth group brought together the deep stations 3 and 4 situated at depths between 58 and $85 \mathrm{~m}$ (group D). Group $A$ and $B$ showed the lowest average values for diversity $(0.73 \pm 0.67)$ and density $(4.80 \pm 3.70$ ind./0.1 $\left.\mathrm{m}^{2}\right)$, respectively, and group $\mathrm{D}$ showed the highest average values for diversity $(1.70 \pm 0.14)$, density $\left(33.00 \pm 30.81\right.$ ind.$\left./ 0.1 \mathrm{~m}^{2}\right)$ and richness $(11$ sps.).

Cluster analysis results for the summer campaign presented 4 groups with a $30 \%$ level of similarity (Fig. 6). These groups showed clear separation regarding depth, as evidenced by ANOSIM. Group A comprises the shallower coastal stations, group B the stations placed at $30 \mathrm{~m}$ depth in the southern part of the area, group $\mathrm{C}$ was located around the $30 \mathrm{~m}$ isobath, but in the central-north area, and group D comprises the deeper stations where cumaceans occurred, at around $50 \mathrm{~m}$ depth. The lowest values for diversity $(1.08 \pm 0.60)$, density $(7.64$ \pm 6.17 ind.$/ 0.1 \mathrm{~m}^{2}$ ), and richness (6 sps.) are found in group A, while group D showed the highest density $\left(78.40 \pm 81.16\right.$ ind. $\left./ 0.1 \mathrm{~m}^{2}\right)$ and higher diversity $(1.46 \pm$ 0.73 ) (Table 5).

Table 3. ANOSIM results for summer (2006) surveys. Group 1 (stations between 10-25m: 1, 5, 7, 9, 13, 16 and 18), Group 2 (stations between 26-40m: 2, 6, 8, 10, 14, 15, 17 and 19), Group 3 (stations between 41-60m: 3, 11 and 20) and Group 4 (stations between 61-100m: 4, 12 and 21). Global R: 0.604 . $(\mathrm{p}<5 \%)$

\begin{tabular}{lcccc}
\hline Survey & Groups & $\begin{array}{c}\text { "R" } \\
\text { Statistic }\end{array}$ & $\begin{array}{c}\text { Significance } \\
\text { Level “p" (\%) }\end{array}$ & $\begin{array}{c}\text { Possible } \\
\text { Permutations }\end{array}$ \\
\hline \hline Summer & 1 e 2 & 0.541 & 0.1 & 145422675 \\
Summer & 1 e 3 & 0.696 & 0.1 & 74613 \\
Summer & 1 e 4 & 0.685 & 0.2 & 74613 \\
Summer & 2 e 3 & 0.467 & 0.1 & 38760 \\
Summer & 2 e 4 & 0.729 & 0.1 & 38760 \\
Summer & 3 e 4 & 0.708 & 0.2 & 462 \\
\hline
\end{tabular}

Table 4. Mean values ( \pm standard deviation) of the communities' structural parameters and abiotic variables on each group formed by the cluster analysis (winter survey 2005)

\begin{tabular}{|c|c|c|c|c|}
\hline \multirow[b]{2}{*}{ Variables } & \multicolumn{4}{|c|}{ Groups } \\
\hline & $\mathbf{A}$ & B & $\mathrm{C}$ & D \\
\hline$\overline{\mathbf{H}^{\prime}}$ & $0.73 \pm 0.67$ & $0.92 \pm 0.56$ & $1.62 \pm 0.52$ & $1.70 \pm 0.14$ \\
\hline $\mathbf{s}$ & 5.00 & 4.00 & 11.00 & 11.00 \\
\hline Density (ind $/ 0.1 \mathrm{~m}^{2}$ ) & $5.60 \pm 4.99$ & $4.80 \pm 3.70$ & $8.50 \pm 5.68$ & $33.00 \pm 30.81$ \\
\hline Dominant Specie & D. sympterygiae & C. reticulata & A. notus & Cumella sp. $n$. \\
\hline Depth (m) & $28.26 \pm 10.06$ & $28.39 \pm 5.55$ & $42.13 \pm 11.16$ & $71.73 \pm 18.96$ \\
\hline Temperature $\left({ }^{\circ} \mathrm{C}\right)$ & $20.95 \pm 0.19$ & $20.92 \pm 0.49$ & $21.09 \pm 0.25$ & $19.02 \pm 2.26$ \\
\hline Salinity & $35.15 \pm 0.41$ & $35.01 \pm 0.25$ & $35.39 \pm 0.55$ & $35.56 \pm 0.45$ \\
\hline Organic Matter (\%) & $1.35 \pm 0.49$ & $1.19 \pm 0.42$ & $1.63 \pm 0.60$ & $2.99 \pm 0.96$ \\
\hline Carbonate (\%) & $4.33 \pm 2.27$ & $4.57 \pm 1.20$ & $11.31 \pm 6.33$ & $14.22 \pm 7.83$ \\
\hline GRN (\%) & $0.30 \pm 0.61$ & $0.01 \pm 0.02$ & $3.52 \pm 4.41$ & $1.91 \pm 2.42$ \\
\hline VCS (\%) & $0.21 \pm 0.19$ & $0.22 \pm 0.24$ & $1.58 \pm 1.34$ & $1.68 \pm 1.95$ \\
\hline CS (\%) & $0.32 \pm 0.29$ & $0.36 \pm 0.29$ & $1.13 \pm 0.51$ & $1.03 \pm 0.93$ \\
\hline MS (\%) & $0.69 \pm 0.43$ & $0.72 \pm 0.41$ & $1.24 \pm 0.46$ & $1.62 \pm 1.39$ \\
\hline FS (\%) & $18.88 \pm 14.12$ & $29.41 \pm 20.91$ & $19.63 \pm 15.47$ & $27.05 \pm 24.10$ \\
\hline VFS (\%) & $73.58 \pm 12.94$ & $67.28 \pm 18.99$ & $63.78 \pm 11.26$ & $53.33 \pm 30.65$ \\
\hline SIL (\%) & $2.08 \pm 1.48$ & $0.42 \pm 0.85$ & $2.98 \pm 2.14$ & $3.34 \pm 2.34$ \\
\hline CL (\%) & $3.95 \pm 2.95$ & $1.27 \pm 2.53$ & $5.94 \pm 4.49$ & $10.05 \pm 2.46$ \\
\hline
\end{tabular}


Table 5. Mean values ( \pm standard deviation) of the communities' structural parameters and abiotic variables on each group formed by the cluster analysis (summer survey 2006).

\begin{tabular}{|c|c|c|c|c|}
\hline Variables & A & B & $\mathrm{C}$ & D \\
\hline$\overline{\mathrm{H}^{\prime}}$ & $1.08 \pm 0.60$ & $1.10 \pm 0.65$ & $1.30 \pm 0.67$ & $1.46 \pm 0.73$ \\
\hline $\mathrm{S}$ & 6.00 & 10.00 & 7.00 & 10.00 \\
\hline Density (ind. $/ 0.1 \mathrm{~m}^{2}$ ) & $7.64 \pm 6.17$ & $8.50 \pm 7.19$ & $13.11 \pm 10.26$ & $78.40 \pm 81.16$ \\
\hline Dominant Specie & D. sympterygiae & O. salinoi & A. notus & A. notus \\
\hline Depth (m) & $16.30 \pm 2.63$ & $32.72 \pm 12.32$ & $30.08 \pm 2.46$ & $54.32 \pm 4.17$ \\
\hline Temperature $\left({ }^{\circ} \mathrm{C}\right)$ & $25.98 \pm 0.65$ & $19.88 \pm 1.98$ & $19.88 \pm 0.69$ & $17.07 \pm 0.21$ \\
\hline Salinity & $34.05 \pm 0.56$ & $35.66 \pm 0.36$ & $35.74 \pm 0.07$ & $35.02 \pm 0.65$ \\
\hline Organic Matter (\%) & $3.89 \pm 2.42$ & $2.29 \pm 2.07$ & $3.64 \pm 3.30$ & $2.30 \pm 0.72$ \\
\hline Carbonate (\%) & $3.82 \pm 1.13$ & $4.63 \pm 2.40$ & $4.71 \pm 1.19$ & $11.44 \pm 3.62$ \\
\hline GRN (\%) & $0.01 \pm 0.01$ & $0.03 \pm 0.05$ & $0.42 \pm 0.74$ & $0.65 \pm 0.78$ \\
\hline VCS $(\%)$ & $0.04 \pm 0.05$ & $0.20 \pm 0.13$ & $0.77 \pm 0.85$ & $0.66 \pm 0.53$ \\
\hline CS $(\%)$ & $0.05 \pm 0.03$ & $0.25 \pm 0.11$ & $0.66 \pm 0.50$ & $0.73 \pm 0.52$ \\
\hline MS (\%) & $0.16 \pm 0.11$ & $0.70 \pm 0.43$ & $0.77 \pm 0.47$ & $0.86 \pm 0.52$ \\
\hline FS (\%) & $4.33 \pm 3.01$ & $27.16 \pm 20.26$ & $22.31 \pm 13.86$ & $9.28 \pm 3.19$ \\
\hline VFS (\%) & $85.10 \pm 10.93$ & $65.27 \pm 14.48$ & $67.85 \pm 8.52$ & $74.77 \pm 8.37$ \\
\hline SIL (\%) & $3.90 \pm 3.27$ & $1.94 \pm 3.04$ & $1.31 \pm 2.11$ & $5.68 \pm 2.62$ \\
\hline CL $(\%)$ & $6.41 \pm 7.74$ & $4.44 \pm 5.32$ & $5.92 \pm 6.38$ & $7.37 \pm 2.53$ \\
\hline
\end{tabular}

Cumacean Assemblages and

Environmental Parameters

The BIOENV analysis, applied simultaneously to the species density data and to environmental variables, showed that the distribution and composition of cumacean assemblages in the winter campaign was better explained by the set of depth variables and percentage of granules $(\rho=0.402)$. Depth alone showed the highest level $(\rho=0.393)$, followed by carbonate $(\rho=0.316)$. For the summer campaign, the highest correlation value was for depth and percentage of coarse sand coupled $(\rho=0.624)$. As in the previous campaign, depth alone presented the highest correlation $(\rho=0.654)$, followed by percentage of coarse sand $(\rho=0.493)$ (Table 6$)$.

Spearman correlation with environmental and biological data in the winter campaign showed diversity positively correlated with granules $(r=0.60)$ and very coarse sand $(r=0.58)$; richness with very coarse sand $(r=0.45)$, and density negatively correlated with very fine sand $(\mathrm{r}=-0.76)$. Regarding the species, a negative correlation was found between Cyclaspis reticulata and the amount of silt $(\mathrm{r}=-0.87)$ and clay $(\mathrm{r}=-0.87)$, and between Cumella sp. A and very fine sand $(\mathrm{r}=-0.62)$. Diastylis sympterygiae correlated positively with diversity $(\mathrm{r}=0.54)$ and salinity $(r=0.49)$. For the summer campaign, diversity showed negative correlation with silt $(\mathrm{r}=-0.46)$ and organic matter $(\mathrm{r}=-0.47)$ and richness showed positive correlation with very fine sand $(r=0.47)$ and negative correlation with silt $(\mathrm{r}=-0.51)$, clay $(\mathrm{r}=-0.47)$ and organic matter $(\mathrm{r}=-0.62)$. Cumella sp. A and Oxyurostylis salinoi correlated positively with pheopigments $(\mathrm{r}=0.51)$ and fine sand $(\mathrm{r}=0.44)$, respectively. Diastylis sympterygiae correlated positively with temperature $(\mathrm{r}=0.54)$ and negatively with salinity $(\mathrm{r}=-0.71)$. All correlations were significant at $\rho<5 \%$.

Table 6. BIOENV results, showing the best correlations between abiotic and biotic matrixes on winter (2005) and summer survey (2006).

\begin{tabular}{cccc}
\hline Survey & $\begin{array}{c}\text { Number of } \\
\text { variables }\end{array}$ & Variables & Correlation ( $\boldsymbol{\rho}$ ) \\
\hline \hline Winter & $\mathbf{1}$ & Depth & 0.393 \\
Winter & $\mathbf{1}$ & Carbonate & 0.316 \\
Winter & $\mathbf{2}$ & Depth, Granule & 0.402 \\
Winter & $\mathbf{2}$ & Granule, Organic matter & 0.391 \\
Summer & $\mathbf{1}$ & Depth & 0.654 \\
Summer & $\mathbf{1}$ & Coarse sand & 0.493 \\
Summer & $\mathbf{2}$ & Depth, Coarse sand & 0.624 \\
Summer & $\mathbf{2}$ & Depth, Very Coarse sand & 0.620 \\
\hline \hline
\end{tabular}

\section{DisCUSSION}

A temporal difference was detected in the total abundance of cumaceans in the present study, with numbers nearly 3 times higher in summer than in the winter. The summertime high abundance could be related to the presence of Anchistylis notus, as the species accounted for more than half the total number of individuals of the campaign. However, temporal differences occurred only at a few stations. As an example, of the total of A. notus obtained in February 2006 about $76 \%$ of the individuals were present at station 20. Therefore, for the major part of the stations no significance in abundance was detected as between seasons. Such behavior presented by cumaceans on the Santos shelf, however, is not the general rule found for the group in other areas of the Brazilian southern 
coast. A remarkable seasonal change in cumacean density has been found on the whole continental shelf off Ubatuba, a region to the north of the present study area, in a depth range similar to that of this present study. The cumaceans there attained, in summer, an average 25 times greater than in the winter $(583$ ind. $/ 0.1 \mathrm{~m}^{2}$ and 23 ind. $/ 0.1 \mathrm{~m}^{2}$, respectively for each season) (SANTOS; PIRES-VANIN, 1999). In studies involving amphipods, another peracarid group, conducted in the same area of the Santos shelf (RODRIGUES; PIRES-VANIN, 2012) and on the nearby São Sebastião shelf (VALÉRIO-BERARDO et al., 2000), a similar, though less marked, difference between summer and winter periods has also been observed. In addition, distinct higher density and biomass have been reported for several other invertebrate groups on the Brazilian southeastern coast (PIRES, 1992; DE LÉO; PIRES-VANIN, 2006). Thus, the dynamics of the cumacean communities indicate the existence of temporal variation in the Santos shelf area, although it is most probably to be related to the behavior and life cycle of just one species, Anchistylis notus.

As regards the density of cumaceans in sedimentary bottoms around the world, high values tend to be found in more eutrophic environments (DAYTON; OLIVER, 1977). On the southern and southeastern Atlantic shelves density usually peaks in summer months and this fact has been attributed to the quantity and quality of organic matter that reaches the bottom at this time of the year (VENTURINI et al., 2011b). Intense eutrophication occurs in the water column during summer caused by the superficial intrusion of SACW on the continental shelf, which favors the increase in biomass and phytoplankton productivity in midwater with the consequent exportation of organic matter to benthic communities (VALÉRIO-BERARDO et al., 2000; VENTURINI et al., 2011a). Due to the intrusion of the cold water mass over the bottom and the heating of the superficial water, a thermocline forms at intermediate depths (CASTRO; MIRANDA, 1998) and as a consequence of the stability of water column, the new production is kept close to the bottom (AIDAR et al., 1993; METZLER et al., 1997).

In addition to eutrophication, the life cycles of the species as well as biological interactions among individuals and communities play an important role in structuring benthic communities. Seasonal reproduction is common among shallow water cumaceans, and may be synchronized with the food input to the bottom due the intensification of primary production (GRANGER et al., 1979). Also, the number of broods per year in cumaceans is probably regulated by the temperature range and food availability, as shown in Corbera et al., 2000.
Cumaceans were poorly represented or even absent at some stations of the Santos shelf. Their absence at coastal stations 5 and 13 in the winter period may be related to sediment turning caused by environmental instability, more specifically by the increasingly strong winds which occur during winter. Cold fronts have been implicated as explaining low macrofauna densities on the inner Brazilian shelf (SOARESGOMES; PIRES-VANIN, 2003; ROSA; BEMVENUTI, 2006) and around the world (POSEY et al., 1996; WATLING; GERKEN, 2005), since they affect sedentary animals and those, such as the cumaceans, which burrow superficially by effectively carrying them out of the area (POSEY, et al., 1996).

Changes in sediments due to erosion and/or deposition may cause the submersion of macrofauna and affect community composition (GRANT, 1983; CONRADI et al., 2000). This situation seems to occur at station 13, located at the mouth of Santos Bay. The area shows intense deposition of sediments originating from the Santos estuarine system (MAHIQUES, et al., 2004) and a great loss of amphipod species (RODRIGUES; PIRES-VANIN, 2012) and polychaetes (M. SHIMABUKURO, IOUSP, pers.comm.) has been observed there. Disruptions of the sediment also cause a reduction of resources and an inhibition of feeding as well as the removal of digger organisms, exposing the fauna to the action of predators, which, consequently, affect the density and diversity of the communities present at a given site (CARTES et al., 2003; LU, 2005).

Coastal regions close to major urban centers are additionally subject to the influence of human activities, such as sewage discharge, estuary contamination by oil spills, and other organic contaminants (MARTINS et al., 2008). In the Santos shelf area, the influence of sewage might be detected near the Bertioga Channel and this is reflected in the distribution of crustaceans (PIRES-VANIN et al., 2008). The authors reported that for samples from station 5, near the Channel opening, the species were absent or extremely reduced ( 3 ind. $/ 0.1 \mathrm{~m}^{2}$ ), which contrasted with data from station 6 , a place with the same salinity but without the influence of the Channel waters. As cumaceans are negative bio indicators (CONLAN et al., 2004), we assume the existence of a trend towards the reduction, or even the elimination, of the population in the presence of contaminants.

Regarding the absence of cumaceans at deep stations 12 and 21, this is most probably related to the high percentage of silt and clay present in the sediments of the area. A similar decline in cumacean density was reported by Martin et al. (2010) in a study carried out in the Persian Gulf region and the authors related the fact to the species' feeding habit. Little is known about the feeding of cumaceans but, despite the species' not presenting a uniform feeding method 
(CORBERA, 2006; MARTIN et al., 2010), it may be assumed that shallow water species consume mainly epipelic food while deep water species feed mostly on pelagic sedimented particles (BLAZEWICZ-PASZKOWYCZ; LOGOWSKI, 2002). Thus, for shallow water species the ideal is that the grains should be of moderate size, neither very coarse nor very fine, allowing their handling by the animals' mouth parts (WIESER, 1956). However, a detailed study of the morphology of the cumaceans' mouthparts is necessary to confirm the feeding habit of those of the Santos shelf.

Five species with high average densities were highly abundant in the area. The high abundance of Diastylis sympterygiae on both campaigns might be related to its euryhaline capacity, since it tolerates salinity ranging from 0 to 34.7 (BEMVENUTI et al., 2005; CRISTALES et al., 2010). The species is capable of tolerating places with high saline instability, as is the case of the Lagoa dos Patos in southern Brazil, it being the only cumacean reported in the area (ROSA; BEMVENUTI, 2007). This capacity is an adaptive advantage for the species, favoring the occupation of estuarine areas, and is exemplified by the southern coastal station 18 of the present study, situated near the mouth of the Peruíbe River, where $D$. sympterygiae presents great numerical dominance. Modlin (1992) considered salinity as an important factor in the structuring of the cumacean community in the coastal waters of the Gulf of Mexico, and discovered that low values of salinity tended to affect macrofauna negatively for most of the species.

Cumaceans were distributed in well-defined vertical zones in the substrate, with a massive concentration in the upper layer $(0-2.5 \mathrm{~cm})$. This reflects the group's lifestyle, by reason of which the organisms remain part of the time partially covered to produce the respiratory and feeding current that depends on water with high oxygen concentration (CORBERA et al., 2002). Generally, higher macrofauna density has been observed in the upper layer of sediments (GAMBI; BUSSOTTI, 1999; FILGUEIRAS et al., 2007). Corbera et al. (2002) pointed out that most species sampled in the Canary Islands were obtained in the upper layer $(0-5 \mathrm{~cm})$ and few organisms, less than $10 \%$, were found in deeper strata. A similar result was reported for amphipods (RODRIGUES; PIRES-VANIN, 2012) and polychaetes (M. SHIMABUKURO, pers. comm.) on the Santos shelf.

Regarding diversity, richness, and density, the values were higher in the area near the 50 meter isobath in both the seasons sampled. Several studies on the diversity of benthic macrofauna of the southwestern Atlantic have reported the occurrence of significantly higher values on the middle shelf
(VENTURINI et al., 2011b; RODRIGUES; PIRESVANIN, 2012), and water column stability and continuous input of phytoplankton through alternating new and regenerated production (AIDAR et al., 1993; METZLER et al., 1997) have been evoked to explain these findings. As has been thoroughly documented, depth is a driving factor for the distribution of marine benthos, as it generates an environmental gradient of temperature, light and velocity of tidal currents and wind, among others (ELLINGSEN, 2002; GRAY; ELLIOTT, 2009). Consequently, the biological variables of the community reflect this gradient. Studies of continental shelves of subtropical and temperate seas around the world have shown that density and diversity tend to increase seawards, since offshore environments are usually more stable and favorable for species diversity enhancement (CARTES; SORBE, 1997; GRAY; ELLIOTT, 2009).

Sediment is among the most important factors related to species settlement in the benthic environment, and frequently best explains the distributional patterns of macrofauna on many continental shelves worldwide (GRAY; ELLIOTT, 2009), consequently, it has been considered a super parameter (MUNIZ; PIRES, 2000). Capítoli \& Bemvenuti $(2004,2006)$ showed the distribution of macrofauna species on a sedimentary and depth gradient on the continental shelf and slope in the southern Atlantic and observed that faunal changes were mainly related to variations in the substrate type. In a study carried out in the North Carolina region, very fine sand and the amount of silt and clay were the most important variables for benthic communities on the inner shelf (WESTON, 1988), and similar patterns occur on other shelves in various oceans (LU, 2005; MARTIN et al., 2010).

Mixed sediments have been reported as most favorable for the occurrence of cumaceans. Fine sand with the addition of small amounts of silt and calcareous fragments constituted the most adequate substratum for the settlement of cumacean communities on the southeastern and southwestern coastal areas of India (RADHADEVI; KURIAN, 1989). The negative correlation found in our study between Cyclaspis reticulata and the amounts of silt and clay, Cumella sp. A and very fine sand, and the positive correlation between Oxyurostylis salinoi and fine sand seem to reinforce the importance of the sediment particle size composition for cumacean occurrence. Morphological characteristics, such as the space between the bristles on the third maxilliped might explain the presence of cumaceans on a given sort of bottom. The third maxilliped has small bristles at its base that work as a fine sieve and, if the orifice between these structures is not fine enough, suspended debris can enter the gill chamber and cause 
asphyxia and death. The size of the space between these setae, therefore, is very likely a determining factor of the type of substrate on which the animal can live (DIXON, 1944). Such a combination of different fine grains, as present at stations 3 and 20, can be specific for the dominance of Anchistylis notus and/or for the high diversity found.

\section{ACKNOWLEDGEMENTS}

The authors are grateful to Prof. Dr. Daniel Roccatagliata for his help with species identification and to the anonymous reviewer for suggestions on the manuscript. Financial support was provided by the Fundação de Amparo à Pesquisa do Estado de São Paulo (FAPESP) to AMSPV (ECOSAN Project, Proc. $\mathrm{n}^{\mathrm{o}}$ 03/09932-1) and to PAC (M.Sc. grant, Proc. $\mathrm{n}^{\mathrm{o}}$ 2008/55842-8).

\section{REFERENCES}

AIDAR, E.; GAETA, S. A.; GIANESELLA-GALVÃO, S. M. F.; KUTNER, M. B. B.; TEIXEIRA, C. Ecossistema costeiro subtropical: nutrientes dissolvidos, fitoplâncton e clorofila $a$ e suas relações com as condições oceanográficas na região de Ubatuba, São Paulo. Publ. Esp. Inst. Oceanogr, São Paulo., v. 10, p. 9-43, 1993.

ALFONSO, M. I.; BANDERA, M. E.; LÓPEZ-GONZÁLEZ, P. J.; GARCÍA-GOMEZ, J. C. The cumacean community associated with a seaweed as a bioindicator of environmental conditions in the Algeciras Bay (Strait of Gibraltar). Cah. Biol. Mar., v. 39, p. 197-205, 1998.

ANGER, K.; VALENTIN, C. In situ studies on the diurnal activity pattern of Diastylis rathkei (Cumacea, Crustacea) and its importance for the 'hyperbenthos'. Helgoländer Wiss. Meer., v. 28, p. 138-144, 1976.

BĂCESCU, M.; PETRESCU, I. A new contribution to the knowledge of the cumaceans (Crustacea, Diastylidae) from the Brazilian waters. Trav. Mus. Hist. Nat. "Grigore Antipa"., v. 31, p. 387-395, 1990.

BĂCESCU, M.; QUEIROZ, E. L. The contribution of Cumacea in the feeding of the Rajidae Sympterygia acuta and S. bonapartei from Rio Grande do Sul-S. Brazil. Trav. Mus. Hist. Nat. "Grigore Antipa"., v. 27, p. 7-18, 1985.

BEMVENUTI, C. E.; ANGONESI, L. G.; GANDRA, M. S. Effects of dredging operations on soft bottom macrofauna in a harbor in the Patos Lagoon estuarine region of southern Brazil. Braz. J. Biol., v. 65, n. 4, p. 573-581, 2005.

BLAZEWICZ-PASZKOWYCZ, M.; LOGOWSK, I. R. Diatoms as food indicator for some Antarctic Cumacea and Tanaidacea (Crustacea). Antarctic Science, v. 14, n. 1, p. 11-15, 2002.

BRUM, I. N. S. Oxyurostylis salinoi sp. n. do litoral brasileiro (Crustacea, Cumacea). Rev. Bras. Biol., v. 26 n. 1, p. 59-67, 1966.

BYERS, S. C.; MILLS, E. L.; STEWART, P. L. A comparison of methods to determining organic carbon in marine sediments, with suggestion for a standard method. Hydrobiologia., v. 58, p. 43-57, 1978.

CAPÍTOLI, R. R.; BEMVENUTI, C. E. Distribuição Batimétrica e Variações de Diversidade dos Macroinvertebrados Bentônicos da Plataforma Continental e Talude Superior no extremo sul do Brasil. Atlântica, Rio Grande., v. 26, p. 27-43, 2004.

CAPÍTOLI, R. R.; BEMVENUTI, C. E. Associações de macroinvertebrados bentônicos de fundos inconsolidados da plataforma continental e talude superior no extremo sul do Brasil. Atlântica, Rio Grande., v. 28, n. 1, p. 4759, 2006.

CARTES, J. E.; JAUME, D.; MADURELL, T. Local changes in the composition and community structure of suprabenthic peracarid crustaceans on the bathyal Mediterranean: influence of environmental factors. Mar. Biol., v. 143, p. 745-758, 2003.

CARTES, J. E.; SORBE, J. C. Bathyal cumaceans of the Catalan Sea (North-western Mediterranean): faunistic composition, diversity and near-bottom distribution along the slope (between 389 and 1859 m). J. Nat. Hist., v. 31, n. 7, p. 1041-1054, 1997.

CASTRO, B. M.; MIRANDA, L. B. de. Physical oceanography of the western atlantic continental shelf located between $4 \mathrm{~N}$ and $34 \mathrm{~S}$. Coastal segment $(4, \mathrm{w})$. In: ROBINSON, A. R.; BRINK, K. H. (Eds.). The Sea. Chapter 8, V 11. John Willay \& Sons Inc, 1998. p. 209251.

CLARKE, K. R.; AINSWORTH, M. A method of linking multivariate community structure to environmental variables. Mar. Ecol. Prog. Ser., v. 92, p. 205-219, 1993

CLARKE, K. R.; WARWICK, R. M. Change in marine communities: an approach to statistical analysis and interpretation. $2^{\mathrm{a}}$ ed. London: PRIMER-E, Plymouth Marine Laboratory, Plymouth, 2001. 144p.

ClARKE, K. R.; GORLEY, R. N. Primer v6: user manual/tutorial. London: PRIMER-E, Plymouth Marine Laboratory, Plymouth, 2006. 190p.

CONLAN, K. E.; KIM, S. L.; LENIHAN, H. S.; OLIVER, J. $\mathrm{S}$. Benthic changes during 10 years of organic enrichment by McMurdo Station, Antartica. Mar. Pollut. Bull., v. 49, p. 43-60, 2004.

CONRADI, M.; LÓPEZ-GONZÁLES, P. J.; CERVERA, J. L.; GARCÍA-GOMEZ, J. C. Seasonality and spatial distribution of peracarids associated with the bryozoan Bugula neritina in Algeciras Bay, Spain. J. Crust. Biol., v. 20 , n. 2, p. $334-349,2000$.

CORBERA, J. A new operculate cumacean genus (Bodotriidae, Vaunthompsoniinae) from deep waters of New Caledonia. Zoosystema, v. 28, n. 2, p. 325-330, 2006

CORBERA, J.; CARDELL, M. J. Cumaceans as indicators of eutrophication on soft bottoms. Sci. Mar., v. 59, p. 6369, 1995.

CORBERA, J.; SAN VICENTE, C.; SORBE, J. C. Smallscale distribution, life cycle and secondary production of Cumopsis goodsir in Creixell Beach (western Mediterranean). J. Mar. Biol. Ass. U.K., v. 80, p. 271$282,2000$.

CORBERA, J.; BRITO, M. C.; NÚÑEZ, J. Interstitial cumaceans from Sandy bottoms and Cymodocea meadows of the Canary Islands. Cah. Biol. Mar., v. 43, p. 63-71, 2002. 
CORBERA, J.; SEGONZAC, M. A new Styloptocuma species (Crustacea, Cumacea) from hydrothermal vent fields of the Lau and North Fiji basins (West Pacific). Zoosystema, v. 32, n. 3, p. 439-447, 2010.

CRISTALES, P. A.; ROCCATAGLIATA, D.; ALBERICO, N. A. A redescription of a common euryhaline cumacean from southern Brazil: Diastylis sympterygiae Băcescu \& Queiroz, 1985 (Crustacea: Cumacea). Zootaxa, v. 2631, p. 19-35, 2010.

DE LÉO, F. C.; PIRES-VANIN, A. M. S. Benthic megafauna communities under the influence of the South Atlantic Central water intrusion onto the Brazilian SE shelf: A comparison between an upwelling and a non-upwelling ecosystem. J. Mar. Syst., v. 60, p. 268-284, 2006.

DAYTON, P. K.; OLIVER J. S. Antarctic soft-bottom benthos in oligotrophic and eutrophic environments. Science, 197(4298), p. 55-58, 1977.

DIXON, A. Y. Notes on certain aspects of the biology of Cumopsis goodsiri (Van Beneden) and some other cumaceans in relation to their environment. J. Mar. Biol. Ass. UK., v. 26, p. 61-71, 1944.

ELLINGSEN, K. E. Soft-sediment benthic biodiversity on the continental shelf in relation to environmental variability. Mar. Ecol. Prog. Ser., v. 232, p. 15-27, 2002.

FILGUEIRAS, V. L.; CAMPO, L. S.; LAVRADO, H. P. FRENSEL, R.; POLLERY, R. Vertical distribution of macrobenthic infauna from the shallow sublittoral zone of Admiralty Bay, King George Island, Antarctica. Polar Biol., v. 30, p. 1439-1447, 2007.

GAMBI, M. C.; BUSSOTTI, S. Composition, abundance and stratification of soft bottom macrobenthos from selected areas of the Ross Sea shelf (Antarctica). Polar Biol., v. 21, p. 347-354, 1999.

GRANGER, D.; BRUNEL, P.; MESSIER, D. Cyclé de développement de Leucon nasica (Crustacea, Cumacea) dans la nappe glaciale circalittorale de la baie des Chaleurs, golfe du Saint-Laurent, em 1968 et 1969. Can. J. Zool., v. 54, p. 59-106, 1979.

GRANT, J. The relative magnitude of biological and physical sediment reworking in an intertidal community. J. Mar. Res., v. 41, p. 673-689, 1983.

GRAY, J. S.; ELLIOTT, M. Ecology of Marine Sediments. From science to Management. $2^{\text {nd }}$ ed. New York: Oxford Univ. Press, 2009. 225 p.

GROSS, M. G. Carbon determination. In: CARVER, R. E. (Ed.). Procedures in sedimentary petrology. $1^{\underline{a}}$ ed. New York: Wiley Insterscience, 1971. p. 573-596.

JONES, N. S. British Cumaceans. Synopses of the British Fauna No. 7. London: Academic Press, 1976. 62p.

LU, L. The relationship between soft-bottom macrobenthic communities and environmental variables in Singaporean waters. Mar. Pollut. Bull., v. 51, p. 1034-1040, 2005.

MACQUART-MOULIN, C. La phase pelagique nocturne des cumacés. J. Plankton Res., v. 13, n. 2, p. 313-337, 1991.

MAHIQUES, M. M.; MISHIMA, Y.; RODRIGUES, M. Characteristics of the sedimentary organic matter on the inner and middle continental shelf between Guanabara Bay and São Francisco do Sul, south-eastern Brazilian margin. Cont. Shelf Res., v. 19, p. 775-798, 1999.

MAHIQUES, M. M.; TESSLER M. G.; CIOTTI, A. M.; SILVEIRA, I. C. A.; SOUSA, S. H. M.; FIGUEIRA, R C. L.; TASSINARI, C. C. G.; FURTADO, V. V.;
PASSOS, R. F. Hydrodynamically- driven patterns of recent sedimentation in the shelf and upper slope off southeast Brazil. Cont. Shelf Res., v. 24, p. 1685-1697, 2004.

MARTIN, D.; FERNANDEZ-ARCAYA, U.; TIRADO, P.; DUTRIEUX, E.; CORBERA, J. Relationships between shallow-water cumacean assemblages and sediment characteristics facing the Iranian coast of the Persian Gulf. J. Mar. Biol. Assoc. UK, v. 90, n. 1, p. 125-134, 2010.

MARTINS, C. C.; FERREIRA, J. A.; TANIGUCHI, S.; MAHIQUES, M. M.; BÍCEGO, M. C.; MONTONE, R. C. Spatial distribution of sedimentary linear alkylbenzenes and faecal steroids of Santos Bay and adjoining continental shelf, SW Atlantic, Brazil: Origin and fate of sewage contamination in the shallow coastal environment. Mar. Pollut. Bull., v. 56, p. 1353-1376, 2008.

METZLER, P. M.; GLIBERT, P. M.; GAETA, S. A.; LUDLAM, J. M. New and regenerated production in the South Atlantic off Brazil. Deep-Sea Res. Pt. I., v. 44, n. 3, p. 363-384, 1997.

MODLIN, R. F. Population structure, distribution, life cycle and reproductive strategy of Spilocuma watlingi Omholt and Heard, 1979 and S. salomani Watling, 1977 (Cumacea: Bodotriidae) from coastal waters of The Gulf of Mexico. Northe. Gulf Sci. v. 12, n. 2, p. 83-91, 1992.

MUNIZ, P.; PIRES, A. M. S. Polychaete associations in a subtropical environment (São Sebastião Channel, Brazil): a structural analysis. P.S.Z.N. Mar. Ecol., v. 21, n.2, p. $145-160,2000$.

PIELOU, E. C. Ecological Diversity. New York: J.W. Ley Ed, 1975. 165p.

PIRES, A. M. S. Structure and dynamics of benthic megafauna on the continental shelf offshore of Ubatuba, southeastern Brazil. Mar. Ecol. Prog. Ser., v. 86, p. 6376, 1992.

PIRES-VANIN, A. M. S.; BROMBERG, S.; FERREIRA, J. A.; RODRIGUES, C. W.; SHIMABUKURU, M. Bentos. Megafauna. Plataforma continental adjacente situada entre São Sebastião e Peruíbe. In: PIRES-VANIN, A. M. S. (Org.). A influência do complexo estuarino da Baixada Santista sobre o ecossistema da plataforma adjacente - ECOSAN. Relatório Técnico, 4v, 2008. p. 462-494.

POSEY, M. H.; LINDBERG, W.; ALPHIN, T.; VOSE, F. Influence of storm disturbance on an offshore benthic community. Bull. Mar. Sci., v. 59, p. 523-529, 1996.

RADHADEVI, A.; KURIAN, C. V. A collection of Cumacea from the south west and south east coasts of India. Zool. Surv. India., v. 121, p. 1-37, 1989.

ROCCATAGLIATA, D. On the genus Anchistylis (Crustacea, Cumacea) from the South-West Atlantic. Cah. Biol. Mar., v. 38, p. 35-42, 1997.

RODRIGUES, C. W.; PIRES-VANIN, A. M. S. Spatiotemporal and functional structure of the Amphipod communities off Santos, southwestern Atlantic. Braz. J. Oceanogr., v. 60, n. 3, p. 421-439, 2012.

ROSA, L. C.; BEMVENUTI, C. E. Temporal variability of the estuarine macrofauna of the Patos Lagoon, Brazil. Ver. Biol. Mar. Oceanogr., v. 41, n. 1, p. 1-9, 2006. 
ROSA, L. C.; BEMVENUTI, C. E. Seria a macrofauna bentônica de fundos não consolidados influenciada pelo aumento na complexidade estrutural do habitat? O caso do estuário da Lagoa dos Patos. Braz. J. Aquat. Sci. Technol., v. 11, n. 1, p. 51-56, 2007.

SANTOS, M. F. L. DOS.; PIRES-VANIN, A. M. S. The Cumacea community of the southeastern Brazilian Continental Shelf: structure and dynamics. Sci. Mar., v. 63, n. 1, p. 15-25, 1999.

SANTOS, S. L.; SIMON, J. L. Marine soft-bottom community establishment following annual defaunation: larval or adult recruitment?. Mar. Ecol. Prog. Ser., v. 2, p.235-241, 1980 .

SHANNON, C.E.; WEAVER, W. The mathematical theory of communication. The University of Ilinois Press, Urbana, 1963. 117p.

SMITH, C. R. Ecology of whale falls at the deep-sea floor. Oceanogr. Mar. Biol., v. 41, p. 311-354, 2003.

SOARES-GOMES, A.; PIRES-VANIN, A. M. S. Padrões de abundância, riqueza e diversidade de moluscos bivalves na plataforma continental ao largo de Ubatuba, São Paulo, Brasil: uma comparação metodológica. Rev. Bras. Zool., v. 20, n. 4, p. 717-725, 2003.

SOKAL, R. R.; ROHLF, F. J. The principles and practice of statistics in biological research. New York: W.H. Freeman, 1995. 776p.

STATSOFT INC. STATISTICA for the Windows Operating System. Release 6. Statsoft, Inc, Tulsa OK, USA, 1995.

SUGUIO, T. Introdução à sedimentologia. São Paulo: Edgard Blucher, EDUSP, 1973. 317p.

SUTHERLAND, T. F.; SHEPERD, C. F.; ELNER, R. W. Predation on meiofaunal and macrofaunal invertebrates by western sandpipers (Calidris mauri): evidence for dual foraging modes. Mar. Biol., v. 137, p. 983-993, 2000
SWAILEH, K. M.; ADELUNG, D. Effect of body size and season on the concentration of $\mathrm{Cu}, \mathrm{Cd}, \mathrm{Pb}$ and $\mathrm{Zn}$ in Diastylis rathkei (Kroyer) (Crustace: Cumacea) from Kiel Bay, Western Baltic. Mar. Pollut. Bull., v. 31, n. 13, p. 103-107, 1995.

VALERIO-BERARDO, M. T.; FLYNN, M. N.; WAKABARA, Y. Structure and dynamics of a shelf amphipod taxocoenosis in southeastern Brazil. B. Mar. Sci., v. 66, n. 1, p. 59-72, 2000.

VENTURINI, N.; PIRES-VANIN, A. M. S.; SALHI, M.; BESSONART, M.; MUNIZ, P. Polychaete response to fresh food supply at organically enriched coastal sites: Repercussion on bioturbation potential and trophic structure. J. Mar. Syst., v. 88, p. 526-541, 2011a.

VENTURINI, N.; SALHI, M.; BESSONART, M.; PIRESVANIN, A. M. S. Fatty acid biomarkers of organic matter sources and early diagenetic signatures in sediments from a coastal upwelling area (south-eastern Brazil). Chem. Ecol., p. 1-18, 2011 b.

WATLING, L.; GERKEN, S. The Cumacea of the Faroe Islands region: water mass relationships and North Atlantic biogeography. BIOFAR Proc., p. 137-149, 2005.

WESTON, D. P. Macrobenthos-sediment relationships on the continental shelf off Cape Hatteras North Carolina. Cont. Shelf Res., v. 8, n. 3, p. 267-286, 1988.

WIESER, W. Factors influencing the choice of substratum in Cumella vulgaris Hart (Crustacea, Cumacea). Limnol. Oceanogr., v. 1, n. 4, p. 274-285, 1956.

(Manuscript received 21 January 2013; revised 18 April 2014; accepted 23 April 2014) 